\title{
Analysis of the species composition of microorganisms in dogs with otomycosis
}

\author{
Maria Deneva ${ }^{1}$, Vladimir Orobets ${ }^{1, *}$, Nadezhda Ozheredova $^{1}$, Elena Svetlakova $^{1}$ and \\ Elena Grudeva $^{1}$ \\ ${ }^{1}$ Stavropol State Agrarian University, 12, Zootekhnicheskiy lane, 355017, Stavropol, Russia
}

\begin{abstract}
Otitis in dogs is a common inflammatory disease that is often complicated by secondary bacterial or yeast infections. In patients with chronic otitis externa, the disease is often caused by various combinations of factors. The object of the study was 237 sick dogs with clinical signs of otitis externa, of various age, sex and breed and a fixed population. Samples of the separated external auditory canal of sick dogs were used as material for mycological and bacteriological research. Material was sown on the surface of elective media. Microorganisms were isolated both in monoculture and bacterial-fungal associations. No microorganisms were detected in $14.7 \%$ of the samples. Yeast-like fungi - in $19.4 \%$ of the samples, bacteria - in $21.5 \%$ / In the etiology of otitis associations of Malassezia and bacteria were established in $44.3 \%$ of the samples. As for species composition of fungi of the genus Malassezia, M. pachydermatis accounted for $72.4 \%$ and $M$. furfur - in $27.6 \%$ of samples. When analyzing the generic and species diversity of microorganisms, the most frequently recorded were coccal forms $(87,0 \%)$, less frequently - enterobacteria, nonfermenting bacteria, streptococci, enterococci and corynebacteria. The results obtained can be used to develop effective tools of treatment for dogs with otitis externa.
\end{abstract}

\section{Introduction}

Published data from research results indicate an increase in the incidence of dogs diagnosed with otitis externa. In the etiology of the disease, a significant role is given to yeast-like fungi of the genus Malassezia. Malassezia pachydermatis is important both in veterinary and human medicine. Its taxonomic status and physiological characteristics are understood better now. Skin disease associated with these lipophilic yeast is now widespread, especially in dogs. Malassezia pachydermatis is a part of the normal inhabitants of healthy skin and mucous membranes of dogs [1,2].

The genus Malassezia currently includes 14 lipophilic yeast species. This species forms the normal microbiota of the skin and ear canal of dogs, cats, and other carnivores, causing dermatitis and external otitis media [3-5].

In patients with chronic otitis externa, the disease is often caused by various combinations of primary, predisposing, and preserving factors [6]. In a study of 200 smear

\footnotetext{
${ }^{*}$ Corresponding author: orobets@yandex.ru
} 
samples taken from the external auditory canal in 100 clinically healthy dogs aged from 2 to 10 years, bacteria, yeast, and mold were isolated in 20 samples. 74 samples contain bacteria and mold. Yeast and mold were isolated in 22 samples. A single microorganism was present in 44 samples. In 40 samples, one or more agents were isolated, with Staphylococcus sp. (59\%), Bacillus sp. (15\%) and M. pachydermatis (14\%) were the most frequently isolated microorganisms [7]. In the middle ear, the bacteria were cultured from 48 percent of the total samples studied and mainly included Escherichia coli (8\%), yeast (8\%), staphylococci (7\%), and Branhamella catarrhalis (7\%) [8,9].

The population of $M$. pachydermatis in dogs can outgrow and act as an opportunistic pathogen, causing dermatitis and otitis externa in animals suffering from atopic dermatitis, diaper rash, endocrinopathy, and primary keratinization defects. These accompanying disease may occur without the need to contribute to the development of a yeast infection [10].

The association of various types of yeast-like fungi of the genus Malassezia with animal diseases has become an important event in veterinary medicine, since skin diseases caused by these organisms are very common in certain kinds of dogs. It has been found recently that fungi of the genus Malassezia can cause systemic infection, especially in newborn animals, and this can also contribute to the development of allergic disorders such as atopic dermatitis [11-13].

According to [14], the most common isolated microorganisms were Staphylococcus intermedius (26.8\%), Pseudomonas aeruginosa (23.2\%), $\beta$-hemolytic Streptococcus (12.8\%), Proteus spp. (11.0\%) and Staphylococcus epidermidis (8,5\%).

Different groups of bacteria were identified in culture media. The most frequent were Pseudomona aeruginosa (22.22\%), Proteus mirabilis (13.89\%), Staphylococcus aureus (12.50\%), Staphylococcus epidermidis (8.33\%), Escherichia coli (5.56\%), and Staphylococcus coagulase - negative (5,56\%). A cytological study showed that Malassezia pachydermatis is present in $69.8 \%$ of the studied cases [15].

In otitis media in associative forms of infection, the pathological process may involve gram-positive cocci, gram-negative rods, such as Pseudomonas, yeast Malassezia pachydermatis [16]. Gram-positive rod-shaped bacteria Corynebacterium spp. were part of mixed microbial populations in 79/81 cultures associated with otitis [17]. The three most common organisms isolated from the horizontal auditory canal and middle ear were Staphylococcus middleus, yeast, and Pseudomonas spp. The difference in total isolates or susceptibility between the horizontal auditory canal and the middle ear was found in 34 $(89.5 \%)$ samples [18]. In cytopathological studies, $36 \%$ of the samples isolated only one organism, of which $21 \%$ were with Malassezia spp. Two microorganisms were present in $23 \%$ of the samples [19].

Malassezia pachydermatis yeast $(56 \%)$ was by far the most common organism in dogs with otitis, then Staphylococcus middleus bacteria (23\%), Pseudomonas aeruginosa (12\%), Proteus spp. (6\%) and Streptococcus canis (5\%) [20].

Ear infections were caused mainly by one type of bacteria $(63.6 \%)$. The most common isolated bacterium was Staphylococcus intermedius (27.7\%), but other important species were also found (Pseudomonas aeruginosa, Streptococcus sp. u Staphylococcus sp) [21]. In studies [22], the most frequently isolated microorganisms in dog otitis were Staphylococcus (58.8\%), Malassezia pachydermatis (30.9\%), Streptococcus canis (29.9\%), Proteus spp. (14.4\%) and E. coli (10,3\%).

Corynebacterium auriscanis was the most common type of coryneform. Other corineform isolates have been identified as Corynebacterium amycolatum, Corynebacterium freneyi, and Arcanobacterium-like species. Corineform bacteria in all cases were isolated together with other bacteria, mainly Staphylococcus pseudintermedius alone or in combination with Malassezia pachydermatis [23]. 
The most commonly isolated staphylococci were Staphylococcus epidermidis, Staphylococcus aureus, Staphylococcus simulans, Staphylococcus haemolyticus, Staphylococcus saprophyticus, and Staphylococcus intermedius [24].

A microbiological study of exudate in 26 dogs with chronic external otitis revealed a high incidence of yeast and Staphylococcus aureus in monoculture or in association. In the spectrum of microscopic yeast-like fungi, there was a clear predominance of the genus Candida (48\% of the total number). Malassezia (Pityrosporum) was present only in 3\% of the isolates. Based on this, we can conclude about the important role of yeast-like fungi in the pathogenesis of external otitis in dogs [25].

Therefore, it is very important to study the species composition of microorganisms in dogs with otomycosis, which can serve as a basis for selecting suitable antimicrobial agents [18], as well as for developing effective means and methods of therapy for this animal pathology.

\section{Materials and methods of the studies}

The study was conducted in the period from 2017 to 2018 in the research and testing laboratory of the Epizootology and Microbiology Department, Veterinary Medicine Faculty of the Stavropol State Agricultural University and in veterinary clinics of the Caucasus Mineral Waters region.

The object of the study was 237 sick dogs with clinical signs of external otitis, of different ages (from 1 month and older than 6 years), sex and breed, and fixed population. Samples of the separated external auditory canal of sick dogs were used as material for mycological and bacteriological research.

Diagnosis of external otitis in dogs was carried out taking into account the characteristic clinical manifestations: inflammatory and proliferative changes in the external auditory meatus; hyperemia, exudate discharge and soreness during palpation of the ear area.

Microbiological studies of secretions from the ears of dogs were carried out in accordance with the relevant generally accepted methods. The studied material was sown on the surface of elective media, which were used as yolk-salt agar for the isolation of staphylococci; sugar agar - for streptococci; Bile Esculin Azide Agar - for enterococci; Saburo agar nutrient medium - for fungi of the genus Malassezia, blood agar - for bacterial associates.

Cultures of microorganisms were incubated in a thermostat at $37^{\circ} \mathrm{C}$ for $18-24$ hours, fungi of the genus Malassezia - at $28^{\circ} \mathrm{C}$ for 48 hours.

The species identity of all isolated bacteria was determined based on the Bergey determinant (J. Hoult, 1997) and the determinant of zoopatogenic microorganisms (M. A. Sidorov, 1995), and the identification of fungi was conducted according the recommendations of A. F. Kuznetsov (2001).

\section{Results}

When studying the etiology of external otitis in dogs, we analyzed the species diversity of microorganisms that were isolated from the external auditory meatus of sick dogs. The analysis of the obtained results indicates that microorganisms were isolated both in monoculture and in bacterial-fungal associations.

As a result of the conducted research, it was found that $14.7 \%$ of the samples did not contain any microorganisms. Yeast-like fungi were isolated in $19.4 \%$ of the samples, bacteria - in $21.5 \%$ of the samples, in the etiology of otitis in dogs, associations of Malassezia and bacteria were established in $44.3 \%$ of the studied samples. 
In the species composition of isolated fungi of the genus Malassezia, M. pachydermatis accounted for $72.4 \%$, and $M$. furfur was recorded in $27.6 \%$ of samples.

When analyzing the generic and species diversity of microorganisms isolated from the external auditory canal of dogs with otitis, the most frequently were recorded coccal forms of the genus Staphylococcus $(87,0 \%)$. The species spectrum of the isolated staphylococci was as follows: Staphylococcus aureus - 38,5\%, Staphylococcus hyicus - 29,6\%, Staphylococcus xylosus - 17,4\% и Staphylococcus intermedius - 1,5\% (table 1).

Table 1. Species composition of microorganisms monocultures of isolated from the external auditory canal of dogs with otitis media.

\begin{tabular}{|l|l|c|}
\hline $\begin{array}{c}\text { Kind of } \\
\text { microorganisms }\end{array}$ & \multicolumn{1}{|c|}{ Spectrum of causative agents } & $\begin{array}{c}\text { Frequency of } \\
\text { occurrence, } \%\end{array}$ \\
\hline \multirow{4}{*}{ Fungi } & Malassezia pachydermatis & 72.4 \\
\cline { 2 - 3 } & Malassezia furfur & 27.6 \\
\hline \multirow{4}{*}{ Microorganisms } & Staphylococcus aureus & 38.5 \\
\cline { 2 - 3 } & Staphylococcus hyicus & 29.6 \\
\cline { 2 - 3 } & Staphylococcus xylosus & 17.4 \\
\cline { 2 - 3 } & Pseudomonas aeruginosa & 2.9 \\
\cline { 2 - 3 } & Proteus vulgaris & 3.6 \\
\cline { 2 - 3 } & Enterococcus spp. & 2.8 \\
\cline { 2 - 3 } & Staphylococcus intermedius & 1.5 \\
\cline { 2 - 3 } & Escherichia coli & 2.1 \\
\cline { 2 - 3 } & Corynebacterium spp. & 1.6 \\
\hline
\end{tabular}

Pseudomonas aeruginosa - was isolated in $2.9 \%$ of samples of sick dogs. Culture of pathogenic bacteria of the Enterobacteriaceae family - Proteus vulgaris - were isolated in $3.6 \%$ of samples, gram-negative Escherichia coli - in $2.1 \%$ of samples. Gram-positive facultative anaerobic bacteria of the genus Enterococcus spp. were isolated in $2.8 \%$ of the samples, and microorganisms of the genus Corynebacterium spp. - in 1.6\%.

In the composition of associations of microorganisms, M. pachydermatis prevailed $59.9 \%$, S. aureus $-34.3 \%$, S. xylosus $-31.4 \%$, and S. coli $-20.1 \%$. M. pachydermatis was primarily associated with S. aureus, S. xylosus, and S. hyicus. M. furfur $-\mathrm{c} \mathrm{S}$. aureus and E. coli (table 2).

Table 2. Species composition of microorganisms associations isolated from the external auditory canal of dogs with otitis.

\begin{tabular}{|l|l|c|}
\hline $\begin{array}{c}\text { Quantitative } \\
\text { composition }\end{array}$ & \multicolumn{1}{|c|}{ Spectrum of causative agents } & $\begin{array}{c}\text { Frequency of } \\
\text { registration, \% }\end{array}$ \\
\hline \multirow{5}{*}{$\begin{array}{l}\text { Two- } \\
\text { component }\end{array}$} & M. pachydermatis+ S. aureus & 14.2 \\
\cline { 2 - 3 } & M. pachydermatis + S. xylosus & 16.2 \\
\cline { 2 - 3 } & M. pachydermatis + S. hyicus & 11.4 \\
\cline { 2 - 3 } & S. aureus + C. koresi & 6.7 \\
\cline { 2 - 3 } & P. aeruginosa + P. vulgaris & 2.9 \\
\cline { 2 - 3 } & S. hyicus + E. coli & 4.8 \\
\cline { 2 - 3 } & S. xylosus + Bacillus spp. & 3.8 \\
\cline { 2 - 3 } & S. hyicus + P. vulgaris & 0.9 \\
\hline \multirow{5}{*}{$\begin{array}{c}\text { Three- } \\
\text { component }\end{array}$} & M. furfur + S. aureus + E. coli & 10.5 \\
\cline { 2 - 3 } & M. pachydermatis + S. xylosus + K. oxytoca & 9.5 \\
\cline { 2 - 3 } & M. pachydermatis + Corynebacterium spp.+ P. vulgaris & 8.6 \\
\cline { 2 - 3 } & M. furfur + Streptococcus spp. + Corynebacterium spp. & 3.8 \\
\cline { 2 - 3 } & S. xylosus + Bacillus spp. + P. vulgaris & 1.9 \\
\cline { 2 - 3 } & S. aureus + E. coli + Bacillus spp. & 2.9 \\
\cline { 2 - 3 } & P. aeruginosa + P. vulgaris + Escherichia coli & 1.9 \\
\hline
\end{tabular}


$\mathrm{n}$ two-component bacterial-fungal associations, together with M. pachydermatis, $\mathrm{S}$. aureus was isolated $-14.2 \%$, S. xylosus $-16.2 \%$, and S. hyicus $-11.4 \%$.

$\mathrm{S}$. hyicus выделяли в сочетании с E. coli в $4,8 \%$, с P. vulgaris $-0,9 \%$ случаев. S. хylosus ассоциировала с Вacillus spp. в 3,8\% исследованных проб.

In three-component bacterial-fungal associations, in addition to fungi, M. pachydermatis in association with S. xylosus and $\mathrm{K}$. oxytoca were found in $9.5 \%$ of samples and Corynebacterium spp., P. vulgaris - 8.6\%, M. furfur was also registered in combination with S. aureus and E. coli in 10.5\% of samples, in combination with Streptococcus spp. and Corynebacterium spp. - in 3,8\%. Three-component bacterial associations were also found S. xylosus, Bacillus spp., P. vulgaris $(1,9 \%)$, S. aureus, E. coli, Bacillus spp. (2,9\%), P. aeruginosa, P. vulgaris, Escherichia coli (1,9\%).

\section{Conclusions}

Thus, as a result of the conducted research, a wide species diversity of microorganisms of bacterial and fungal etiology were isolated from the external auditory meatus of dogs with otitis. It was found that the maximum frequency of secretions are fungi of the genus Malassezia-74.2\% and cocci belonging to the genus Staphylococcus $82.8 \%$. Less frequently, enterobacteria, non-fermenting bacteria, streptococci, enterococci, and corynebacteria were isolated in animals suffering from otitis media. The obtained results of the analysis of the species composition of microorganisms in dogs with otitis media of bacterial and fungal etiology will help to develop effective tools and treatment schemes for this pathology.

\section{References}

1. F. J. Cabañes, PLoS Pathog 10, e1003892 (2014)

2. K. Moriello, K. Coyner, S. Vet Dermatol. 28(3) (2017) doi: 10.1111/vde.12440

3. G. Gaitanis, P. Magiatis, M. Hantschke, I. Bassukas, A. Velegraki, Clin. Microbiol. Rev. 25, 106-41 (2012) doi: 10.1128/CMR.00021-11

4. C. Saunders, A. Scheynius, J. Heitman, PLoSPathog 8 (2012) doi: e1002701

5. A. Tragiannidis, G. Bisping, G. Koehler, A. Groll. Mycoses 53, 187-95 (2010) doi 10.1111/j.1439-0507.2009.01814.x/

6. L. Puig, G. Castellá, F.J. Cabañes, Mycopathologia 181(9-10), 681-8 (2016) doi: 10.1007/s11046-016-0026-3

7. O. Keskin, O.Y. Tel, N.B. Arserim Kaya, Journal of Animal and Veterinary Advances 9(3), 496-500 (2010) doi: 10.3923/javaa.2010.496.500

8. G. Zur, B. Lifshitz, T. Bdolah-Abram, Small Anim Pract. 52(5), 254-8 (2011) doi: 10.1111/j.1748-5827.2011.01058.x

9. T. Nuttall, In Practice 38, 17-21 (2016) doi: 10.1136/inp.i1951

10. T. Boekhout, E. Guého-Kellermann, P. Mayser, A. Velegraki, Springer Heidelberg Dordrecht London New York. Library of Congress doi: 10.1007/978-3-642-03616-3

11. R. Bond, D.O. Morris, Jacques Guillot et al., Veterinary Dermatology. 31(1), 28-74 (2020) https://doi.org/10.1111/vde.12809/

12. R.J. Hay, G. Midgley, Medicine Published (2010) doi:10.1007/978-3-642-03616-3_1

13. N. Choi Heather, D. Edginton, C.E. Griffin, C. John, Veterinary Dermatology 29(5), 413-e136 (2018) https://doi.org/10.1111/vde.12664 
14. C. Bourély, G. Cazeau, N. Jarrige, A. Leblond, J.Y. Madec, M. Haenni, E. Gay, Epidemiology and Infection 147 (2019) doi: 10.1017/S0950268818003278

15. A. Lupo, M. Haenni, J.-Y. Madec, Antimicrobial Resistance in Bacteria from Livestock and Companion Animals, 377-393 (2018)

16. N. Barnard, A. Foster, In Practice 39(9), 386-398 (2017) doi: 10.1136/inp.j892

17. K. Henneveld, R.A.W. Rosychuk, F.J. Olea-Popelka, D.R. Hyatt, Journal of the American Animal Hospital Association 48(5), 320-326 (2012) doi: 10.5326/JAAHAMS-5791

18. G. Zur, B. Lifshitz, T. Bdolah-Abram, Small Anim Pract. 52(5), 254-8 (2011) doi: 10.1111/j.1748-5827.2011.01058.x

19. C.A. Graham-Mize, E.J. Rosser Jr., Journal of the American Animal Hospital Association 40(2), 102-108 (2014) doi: 10.5326/0400102

20. D. Scott, W.H. Miller, Equine Dermatology (Elsevier Saunders, St Louis, 2011)

21. Ch.R. Sánchez, E.S. Calle, P.N. Falcón, J.C. Pinto, Revista de Investigaciones Veterinarias del Peru 22(2), 161-166 (2011) ISSN: 16823419

22. D.L. Bugden, Identification and antibiotic susceptibility of bacterial isolates from dogs with otitis externa in Australia 91(1-2), $43-46$ (2013) doi.org/10.1111/avj.12007

23. B. Aalbæk, D.A. Bemis, M. Schjærff, L.A Frank, L. Guardabassi, Veterinary Microbiology 145(3-4), 292-298 (2010). doi: 10.1016/j.vetmic.2010.03.032.

24. K. Becker, C. Heilmann, G. Peters, American Society for Microbiology Journals 2, (2014) doi.org/10.1128/CMR.00109-13

25. E. de Bona, S. Ubiratan Paz Telesca, A. Meneghello Fuentefria, Rev. MVZ Cordoba 17(2) (2012) ISSN 0122-0268 